\title{
Optimal Portfolio under Non-Extensive Statistical Mechanics and Value-at-Risk Constraints
}

\author{
PAN ZHAO ${ }^{a, b}$, JIXIA WANG ${ }^{c, *}$ AND YU SONG ${ }^{d}$ \\ ${ }^{a}$ College of Finance and Mathematics, West Anhui University, Lu'an, Anhui, China \\ ${ }^{b}$ Financial Risk Intelligent Control and Prevention Institute of West Anhui University, Lu'an, Anhui, China \\ ${ }^{c}$ School of Mathematics and Information Sciences, Henan Normal University, Xinxiang, Henan, China \\ ${ }^{d}$ School of Economics and Management, Nanjing University of Science and Technology, Nanjing, Jiangsu, China
}

(Received June 27, 2017)

In this study, we consider the optimal portfolio selection problem with a value-at-risk constraint in the nonextensive statistical mechanics framework. We propose a portfolio selection model, which is suitable not only for normal return distributions, but also for non-normal return distributions. Using Chinese stock data, under the normal and $q$-Gaussian return distributions, we provide empirical results. The results indicate that portfolio selections under the $q$-Gaussian return distributions are considerably different from those under the normal return distributions. Moreover, by using the $q$-Gaussian distribution, the underestimated portfolio risk can be effectively avoided.

DOI: 10.12693/APhysPolA.133.1170

PACS/topics: Tsallis entropy, $q$-Gaussian distribution, value-at-risk, optimal portfolio

\section{Introduction}

The optimal portfolio problem is a crucial issue in finance. In 1952, Markowitz first provided the meanvariance approach for optimal portfolio allocation problem [1]. In the mean-variance model, the risk is defined as the variation of portfolio returns, which implies that agents weigh the probability of negative yields equally to positive yields. However, investors usually treat gains and losses asymmetrically. Recently, some methods measuring the downside risk have been proposed, including semi-variance $[2,3]$, lower partial moment $[4,5]$, lower partial standard deviation [6], value-at-risk (VaR) [7-9] and conditional tail expectation $[10,11]$. Among them, the value-at-risk is one of the most popular measuring risk methods in the practice of risk management. It is defined as the maximum expected loss given a confidence level over a fixed time horizon. For example, a VaR with $1 \%$ tolerance level for a 5 -day holding period, means that the maximum loss incurred during 5 days should exceed the VaR only once in every 100 cases. In practice, agents not only pursue maximum profit but also need to take into account controlling or limiting the amount of risk. Some methods have been introduced to discuss the meanVaR optimal portfolio, in which the VaR is used as a risk constraint imposed on the problem of maximizing the mean of portfolio yields [12]. However, it is in the case that the returns distributions are normal. Several empirical results have indicated that the returns of financial quantities have fat-tail characteristics [13-15].

The Tsallis statistics is a generalization of Boltzmann-

*corresponding author; e-mail: 18005643721@163.com
Gibbs statistics. In 1988, it was proposed by Tsallis with the motivation of providing a statistical framework for far-from-equilibrium complex systems [16]. Recently, the Tsallis statistics mechanics has been successfully applied to study the fluctuations of financial markets. For example, Rak et al. and Bil et al. studied fluctuations of the Polish stock market using the Tsallis statistical mechanics $[17,18]$. Borland developed an option pricing model in the framework of the Tsallis non-extensive statistical mechanics and found that the model can effectively reduce the volatility smile [19]. Kozaki and Sato applied the Tsallis statistical mechanics to stock market and studied the problem of portfolio risk management [20]. Namaki et al. proposed a Tsallis non-extensive statistical approach for detecting crises of emerging and mature markets and found that the index $q$ in the crise period is much greater than that in the other period [21]. Multiple studies have shown that the non-extensive statistical mechanics is a good tool in the financial field [22-25].

In this paper, we propose an optimal portfolio selection model in the framework of Tsallis non-extensive statistical mechanics and imposing value-at-risk constraints.

This article is structured as follows. In Sect. 2, we shall give a brief explanation of Tsallis statistics and $q$ Gaussian distributions. In Sect. 3, we shall propose an optimal portfolio selection model, which maximizes expected return subject to a value-at-risk constraint. Moreover, our model is suitable not only for the normal return distributions but also for non-normal distributions. In Sect. 4, we shall compare the differences of the optimal portfolio strategies under $q$-Gaussian returns distributions and normal returns distributions using Chinese stock market data. Empirical results will be presented. In the final section, we shall summarize the paper. 


\section{Tsallis statistics and $q$-Gaussian distribution}

The Tsallis entropy is a generalization of the Shannon entropy, which considers multi-fractal structures and long-range interactions of systems [26]. It is defined as:

$$
\mathrm{d} S_{q}=\frac{1-\int P(x)^{q} \mathrm{~d} x}{q-1}
$$

under constraints

$$
\begin{aligned}
& \int P(x) \mathrm{d} x=1, \\
& \frac{\int P(x)^{q} x^{2} \mathrm{~d} x}{\int P(x)^{q} \mathrm{~d} x}=\sigma^{2}
\end{aligned}
$$

yields a $q$-Gaussian probability density function

$$
P(x)=\frac{1}{z_{q}}\left(1-\beta_{q}(1-q) x^{2}\right)^{\frac{1}{1-q}},
$$

where

$$
\begin{aligned}
& z_{q}=((2-q)(3-q) c)^{\frac{1}{3-q}} \\
& \beta_{q}=c^{\frac{1-q}{3-q}}((2-q)(3-q))^{\frac{-2}{3-q}} \\
& c=\frac{\pi}{q-1} \frac{\Gamma^{2}\left(\frac{1}{q-1}-\frac{1}{2}\right)}{\Gamma^{2}\left(\frac{1}{q-1}\right)} .
\end{aligned}
$$

$\Gamma(x)$ is gamma function defined by:

$$
\Gamma(x)=\int_{0}^{+\infty} t^{x-1} \mathrm{e}^{-t} \mathrm{~d} t .
$$

Several results have shown that the $q$-Gaussian distributions can fit the fat tails of financial quantities quite well [27-31]. For $q=1$, the $q$-Gaussian distribution is equivalent to a Gaussian distribution. However, for $q>1$, the $q$-Gaussian distribution has the power-law characteristic and exhibits fat tails.

In this paper, we will employ the $q$-Gaussian distribution derived by using the non-extensive statistics mechanics to fit empirical distributions of returns.

\section{Portfolio selection model with value-at-risk constraint}

In this section, we shall present a portfolio selection model with a value-at-risk constraint. That is, we use value-at-risk as the measure for risk and derive an optimal portfolio in the case that the maximum expected loss would not exceed the value-at-risk at a given confidence level.

We consider a financial model consisting of a financing bank account and $n$ risky assets stocks. Suppose that an agent has the initial capital $W_{0}$ to be invested over a finite investment horizon $[0, T]$ and $B$ is the amount borrowed from the bank, $r_{f}$ is a borrowing rate, $w_{i}$ is the proportion invested in the $i$-th stock and $\sum_{i=1}^{n} w_{i}=1$. $P_{i, t}$ is the price of the $i$-th stock at the time $t$. Hence, the initial portfolio satisfies

$$
W_{0}+B=\sum_{i=1}^{n} w_{i} P_{i, 0} .
$$

According to the definition of value-at-risk, at a given confidence level $\alpha$, the agent needs to select a portfolio to make the final wealth satisfy

$$
\operatorname{Prob}\left\{W_{0}-W_{T} \leq \operatorname{VaR}\right\}=\alpha .
$$

Suppose that $r_{w}$ is the total expected return of a portfolio $w$ over a finite investment horizon $[0, T]$. Then, on the portfolio $w$, at the end of the investment horizon $T$, the expected wealth becomes

$$
\begin{aligned}
E & {\left[W_{T, w}\right]=\left(W_{0}+B\right)\left(1+r_{w}\right)-B\left(1+r_{f}\right) } \\
& =W_{0}\left(1+r_{w}\right)+B\left(r_{w}-r_{f}\right) .
\end{aligned}
$$

In Eq. (11), replacing $B$ with Eq. (9) we obtain

$$
\begin{aligned}
E[ & \left.W_{T, w}\right]=W_{0}\left(1+r_{w}\right)+\left(\sum_{i=1}^{n} w_{i} P_{i, 0}-W_{0}\right)\left(r_{w}-r_{f}\right) \\
& =W_{0}+W_{0} r_{w}+\sum_{i=1}^{n} w_{i} P_{i, 0}\left(r_{w}-r_{f}\right)-W_{0} r_{w}+W_{0} r_{f} \\
& =W_{0}\left(1+r_{f}\right)+\sum_{i=1}^{n} w_{i} P_{i, 0}\left(r_{w}-r_{f}\right) .
\end{aligned}
$$

Equation (10) substituted with Eq. (12) becomes

$$
\operatorname{Prob}\left(r_{w} \geq-\frac{V a R+W_{0} r_{f}}{\sum_{i=1}^{n} w_{i} P_{i, 0}}+r_{f}\right)=\alpha .
$$

Now, we employ the $q$-Gaussian distribution to fit the expected return distribution for the portfolio $w$. Let $q_{\alpha, w}$ denote the quantile corresponding to probability $\alpha$ of the $q$-Gaussian distribution for the portfolio $w$. Then, Eq. (13) can be written as

$$
q_{1-\alpha, w}=-\frac{V a R+W_{0} r_{f}}{\sum_{i=1}^{n} w_{i} P_{i, 0}}+r_{f},
$$

that is

$$
\sum_{i=1}^{n} w_{i} P_{i, 0}=\frac{V a R+W_{0} r_{f}}{r_{f}-q_{1-\alpha, w}} .
$$

Equation (12) substituted with Eq. (15) becomes

$$
E\left[W_{T, w}\right]=W_{0}\left(1+r_{f}\right)+\frac{V a R+W_{0} r_{f}}{r_{f}-q_{1-\alpha, w}}\left(r_{w}-r_{f}\right) .
$$

Equation (16) divided by $W_{0}$ becomes

$$
E\left[\frac{W_{T, w}}{W_{0}}\right]=\left(1+r_{f}\right)+\frac{V a R+W_{0} r_{f}}{W_{0}\left(r_{f}-q_{1-\alpha, w}\right)}\left(r_{w}-r_{f}\right) .
$$

We know that an agent hopes to find a portfolio $w^{*}$, which can maximize the final wealth. That is, we need select a portfolio strategy to maximize Eq. (17). Since the $\mathrm{VaR}$ is the biggest loss given by the investor and $r_{f}$ is a constant, maximizing Eq. (17) can be written as

$$
\max _{w}=\frac{r_{w}-r_{f}}{r_{f}-q_{1-\alpha, w}}, \quad \text { if } \quad \sum_{i=1}^{n} w_{i}=1 .
$$

From Eq. (18), we can note that initial wealth $W_{0}$ and borrowed $B$ do not affect the selection of the optimal portfolio. 


\section{Empirical results}

To test our model, we randomly select four stocks in Chinese stock market and employ daily data from 20132-1 until 2014-9-9. Let borrowing rate $r_{f}=0.06$.

Table I gives the summary statistics for the four stocks returns. The table shows that the returns of the four stocks have significant kurtosis and we have enough evidences to reject the null hypothesis. Hence, there will be great deviations if we use the normal distribution to fit the empirical distribution of the returns.

Summary statistics of four stocks returns

TABLE I

\begin{tabular}{l|c|c|c|c}
\hline \hline stock code & 600136 & 600624 & 600088 & 600639 \\
mean & 0.0016 & 0.0014 & 0.0001 & 0.0008 \\
standard deviation & 0.0270 & 0.0320 & 0.0269 & 0.0220 \\
skewness & -0.0040 & -0.2301 & 0.0645 & 0.3511 \\
kurtosis & 5.7746 & 3.7949 & 4.0534 & 7.8335 \\
Kolmogorov-Smirnov & 1 & 1 & 1 & 1
\end{tabular}

Table II shows the changes of the optimal portfolio allocations at the different confidence levels of VaR constraints under the assumption of the $q$-Gaussian distribution. The VaRs for $1 \$$ held in the portfolios are given in the final column. We can find that the allocations and VaRs of the four stocks are significantly different at different confidence levels. For example, at the confidence level $\alpha=0.95$, the return of the portfolio is maximum at the allocation of $37.05 \%$ stock $600136,2.30 \%$ stock $600624,7.78 \%$ stock 600088 and $52.87 \%$ stock 600639 and the VaR of the optimal portfolio is -2.2965 . However, at the confidence level $\alpha=0.99$, the allocation is $41.21 \%$ stock $600136,9.01 \%$ stock $600624,4.08 \%$ stock 600088 and $45.70 \%$ stock 600639 and the VaR is -4.0491 . Moreover, the smaller tolerance level $1-\alpha$ then the greater return and absolute $\mathrm{VaR}$ of the optimal portfolio.

TABLE II

Optimal portfolios under the $q$-Gaussian distribution

\begin{tabular}{c|c|c|c|c|c|c}
\hline \hline $\begin{array}{c}\text { Confidence } \\
\text { level }\end{array}$ & 600136 & 600624 & 600088 & 600639 & $\begin{array}{c}\text { Portfolio } \\
\text { return }\end{array}$ & $\begin{array}{c}\text { Portfolio } \\
\text { VaR }\end{array}$ \\
\hline 0.95 & 0.3705 & 0.0230 & 0.0778 & 0.5287 & 0.4305 & -2.2965 \\
0.96 & 0.3767 & 0.0548 & 0.0503 & 0.5182 & 0.4355 & -2.3850 \\
0.97 & 0.3852 & 0.0587 & 0.0486 & 0.5075 & 0.4548 & -2.6229 \\
0.98 & 0.3967 & 0.0672 & 0.0472 & 0.4889 & 0.4624 & -3.1061 \\
0.99 & 0.4121 & 0.0901 & 0.0408 & 0.4570 & 0.4772 & -4.0491
\end{tabular}

Table III shows the differences of the optimal portfolio allocations at the diverse confidence levels of VaR constraints under the normal distribution. We can obtain that Table III has the similar law with Table II. However, there are several differences between them. At the same confidence level $\alpha$, firstly, the optimal portfolio allocation in Table III is different from that in Table II. Secondly, the absolute VaR of the optimal portfolio is greater under the $q$-Gaussian distribution than that under the normal distribution, that is to say the portfolio risk is underestimated in the case that yields follow a normal distribution.

TABLE III

Optimal portfolios under the normal distribution

\begin{tabular}{c|c|c|c|c|c|c}
\hline $\begin{array}{c}\text { Confidence } \\
\text { level }\end{array}$ & 600136 & 600624 & 600088 & 600639 & $\begin{array}{c}\text { Portfolio } \\
\text { return }\end{array}$ & $\begin{array}{c}\text { Portfolio } \\
\text { VaR }\end{array}$ \\
\hline 0.95 & 0.2081 & 0.3186 & 0.1710 & 0.3023 & 0.4337 & -2.2710 \\
0.96 & 0.3258 & 0.2010 & 0.1602 & 0.3130 & 0.4468 & -2.3393 \\
0.97 & 0.3556 & 0.2211 & 0.1100 & 0.3133 & 0.4776 & -2.6095 \\
0.98 & 0.3665 & 0.2700 & 0.1305 & 0.2330 & 0.4918 & -2.9729 \\
0.99 & 0.3675 & 0.3221 & 0.1090 & 0.2014 & 0.5138 & -3.5625
\end{tabular}

\section{Summary}

In this paper, we proposed an optimal portfolio model with the value-at-risk constraint. Moreover, we considered the fat-tail characteristics of stock returns under the Tsallis statistical mechanics framework. the empirical results indicated that Tsallis statistical mechanics can be applied to the financial market well. Compared with the normal distribution, the optimal portfolio selection employed the $q$-Gaussian distribution can effectively avoid underestimating the risk.

\section{Acknowledgments}

We would like to thank the referees for helpful comments. We wish to acknowledge financial support from the National Natural Science Foundation of China (Project No. 71302036), the National Natural Science Foundation of China (Project No. U1504701), the Natural Science Key Foundation of the Education Department of Anhui Province of China (Project No. KJ2017A402), the Natural Science Foundation of Anhui Province of China (Project No. 1808085MG224), the Excellent Youth Key Foundation of the Education Department of Anhui Province of China (Project No. gxyqZD2016240), the Soft Science Foundation of Anhui Province of China (Project No. 1607a0202027), the Natural Science Key Foundation of West Anhui University of China (Project No. WXZR201702) and the Humanities and Social Sciences Key Foundation of the Education Department of Anhui Province of China (Project No. SK2016A0971).

\section{References}

[1] H. Markowitz, J. Finance 7, 77 (1952).

[2] W.W. Hogar, J.M. Warren, J. Financial Quantit. Anal. 9, 1 (1974).

[3] Y. Sangchin, L. Tingli, C. Tunjen, C. Kuangjung, Expert Syst. Appl. 38, 2292 (2011).

[4] D.N. Nawrocki, Int. Rev. Financial Anal. 1, 195 (1992).

[5] D. Cumova, D. Nawrocki, J. Econom. Busin. 71, 68 (2014).

[6] A. Frank, S.R. Meer, J. Portfolio Management 17, 27 (1991). 
[7] P. Jorion, VaR-at-Risk: The New Benchmark for Controlling Derivatives Risk, McGraw-Hill, New York 1997.

[8] S. Basak, A. Shapiro, Rev. Financial Stud. 14, 371 (2001).

[9] C. Wei, T. Shaohua, Y. Dongqing, Expert Syst. Appl. 38, 64 (2011).

[10] A.V. Asimit, E. Furman, Q. Tang, R. Vernic, Insur. Math. Econom. 49, 537 (2011).

[11] L. Hua, H. Joe, Insur. Math. Econom. 49, 537 (2011).

[12] G.J. Alexander, A.M. Baptista, J. Econom. Dynam. Contr. 26, 1159 (2002).

[13] T. Lux, J. Econom. Behav. Organiz. 33, 143 (1998).

[14] Z. Yu, Z. Jianwei, W. Zhengxing, Physica 33, 734 (2004).

[15] J. Kittiakarasakun, Y. Tse, Int. Rev. Econom. Finance 20, 430 (2011).

[16] C. Tsallis, J. Statist. Phys. 52, 479 (1988).

[17] R. Rak, S. Drożdż, J. Kwapień, Physica A 374, 315 (2007).

[18] Ł. Bil, D. Grech, E. Podhajska, Acta Phys. Pol. A 129, 986 (2016).

[19] L. Borland, Quantit. Finance 2, 415 (2002).
[20] M. Kozaki, A.H. Sato, Physica A 387, 1225 (2008).

[21] A. Namaki, Z. Lai, G.R. Jafari, R. Raei, R. Tehrani, Physica A 392, 3039 (2013).

[22] C. Tsallis, C. Anteneodo, L. Borland , R. Osorio, Physica A Statist. Mech. Its Appl. 324, 89 (2003).

[23] A.A.G. Cortines, R. Riera, Physica A Statist. Mech. Its Appl. 377, 181 (2006).

[24] J. Ruseckas, V. Gontis, B. Kaulakys, Adv. Complex Syst. 15, 031102 (2012).

[25] C. Tsallis, Entropy 19, 457 (2017).

[26] N. Gradojevic, R. Gencay, Econom. Lett. 100, 27 (2008).

[27] L. Borland, J.P. Bouchaud, Quantit. Finance 4, 499 (2004).

[28] N. Gradojevic, R. Gencay, Signal Process. Mag. 28, 116 (2011).

[29] S.M.D. Queiros, C. Tsallis, Europhys. Lett. 69, 893 (2005).

[30] M. Vellekoop, H. Nieuwenhuis, Quantit. Finance 7, 563 (2007).

[31] S.M.D. Queiros, Quantit. Finance 5, 475 (2005). 